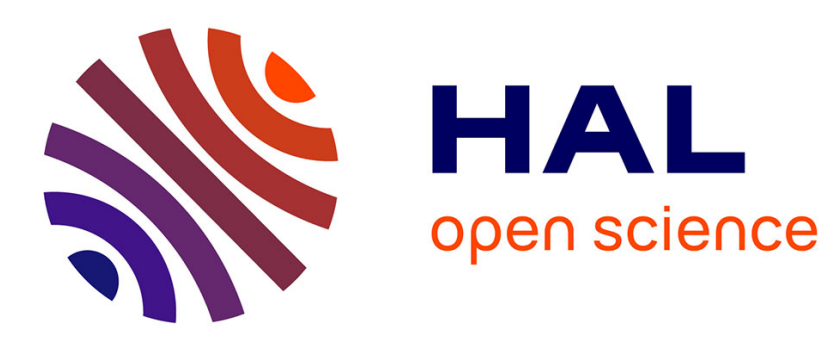

\title{
Étude d'un transducteur électro-optique à cristal liquide pour l'affichage d'hologrammes acoustiques
}

\author{
A. Dmitrieff, J. Perrin, B. Richard, W. Urbach
}

\section{To cite this version:}

A. Dmitrieff, J. Perrin, B. Richard, W. Urbach. Étude d'un transducteur électro-optique à cristal liquide pour l'affichage d'hologrammes acoustiques. Revue de Physique Appliquée, 1976, 11 (4), pp.523-526. 10.1051/rphysap:01976001104052300 . jpa-00244086

\section{HAL Id: jpa-00244086 https://hal.science/jpa-00244086}

Submitted on 1 Jan 1976

HAL is a multi-disciplinary open access archive for the deposit and dissemination of scientific research documents, whether they are published or not. The documents may come from teaching and research institutions in France or abroad, or from public or private research centers.
L'archive ouverte pluridisciplinaire HAL, est destinée au dépôt et à la diffusion de documents scientifiques de niveau recherche, publiés ou non, émanant des établissements d'enseignement et de recherche français ou étrangers, des laboratoires publics ou privés. 


\title{
ÉTUDE D'UN TRANSDUCTEUR ÉLECTRO-OPTIQUE A CRISTAL LIQUIDE POUR L'AFFICHAGE D'HOLOGRAMMES ACOUSTIQUES (*)
}

\author{
A. DMITRIEFF, J. PERRIN, B. RICHARD et W. URBACH \\ Laboratoire de Biophysique (**), C. H. U. Cochin, 24, rue du faubourg Saint-Jacques, 75014 Paris, France
}

(Reçu le 26 septembre 1975, accepté le 16 mars 1976)

\begin{abstract}
Résumé. - L'affichage d'un hologramme sur une matrice à cristal liquide pose des problèmes particuliers. L'image formée doit être exempte de scintillement et de dimensions aussi faibles que possible. La petite taille de la matrice soulève des difficultés par suite de l'existence d'effets de bord et d'interactions entre électrodes. Nous avons donc cherché à déterminer les conditions géométriques favorables à cette application.
\end{abstract}

\begin{abstract}
Displaying a hologram on a liquid crystal matrix raises some specific problems. The picture must be free from scintillations and as small as possible. As a result of the small size of the matrix, side effects and interactions between electrodes are observed. We report studies designed to ascertain the optimum geometrical conditions for this application.
\end{abstract}

Introduction. - Les méthodes d'Imagerie Médicale par les ultrasons suscitent un intérêt croissant en particulier à cause de leur innocuité. L'holographie acoustique pourrait prendre bonne place parmi ces procédés.

La méthode la plus classique consiste à former le hologramme acoustique sur la surface libre d'un liquide. La reconstitution s'opère en temps réel en éclairant le hologramme par réflexion avec un faisceau de lumière cohérente [1]. Mais la grande différence entre les longueurs d'onde acoustique et optique entraîne une déformation de l'image lors de la reconstitution [2].

Dans d'autres méthodes, le hologramme est détecté par balayage ; l'énergie est convertie sous forme lumineuse et enregistrée sur un film photographique.

P. Alais emploie une matrice de transducteurs acoustoélectriques de type électrostatique $(256 \times 256)$ [3]. Dans ce système, les signaux acoustiques reçus sont traduits en signaux électriques. Ceux-ci sont amplifiés et mélangés avec le signal de référence qui est le signal électrique excitant le transducteur émetteur. On a ainsi l'information holographique. Un dispositif électronique interroge séquentiellement les transducteurs de la matrice ce qui permet d'obtenir un signal video classique. Le hologramme complet est formé en $50 \mathrm{~ms}$, permettant le temps réel. La reconstitution optique nécessite de passer par l'intermédiaire d'une photographie de l'écran. On perd donc le temps réel.

(*) Sup. par D. G. R. S. T., contrat GB/P 4306.

(**) Associé au C. N. R. S., E. R. A. 498.
La matrice à cristal liquide étudiée, commandée par le transducteur constituerait un hologramme de phase reproduisant le hologramme acoustique et serait utilisée directement pour la reconstitution en temps réel. $\mathrm{La}$ taille réduite de la matrice diminuerait les distorsions d'image lors de la reconstitution $[4,5]$.

En plus des dimensions réduites de la partie active de la cellule, l'utilisation d'un affichage en tant qu'hologramme présente une grande différence par rapport à l'inscription d'une image classique. En effet un imageur met souvent à profit la rémanence du récepteur (par exemple la persistance rétinienne de l'œil) pour intégrer le balayage de l'image. Dans le cas du hologramme ceci n'est point possible, l'affichage doit être réalisé intégralement sans fluctuations notables.

Le mode biréfringent que nous avons choisi consiste à commander le basculement des molécules d'un nématique, initialement orienté perpendiculairement aux lames, par un champ électrique. Le basculement provoque une modification du déphasage optique introduit par la lame de cristal liquide (C. L.). Ce mode permet d'obtenir une bonne résolution et c'est celui qui offre la meilleure capacité de multiplexage donc qui permet d'afficher le plus grand nombre de points [6-10].

1. La cellule à cristal liquide. - La cellule est constituée par deux lames de verre recouvertes d'une couche conductrice transparente d'oxyde d'indium entre lesquelles est introduit le cristal liquide.

Le C. L. utilisé est nématique à température ambiante et possède une anisotropie diélectrique négative (mélange MBBA EBBA). 
Les molécules allongées du C. L. doivent initialement être disposées perpendiculairement aux plaques de verre (configuration homéotrope). Pour obtenir cette orientation, les lames parfaitement propres sont immergées dans une solution aqueuse d'un surfactant (cétyl trimétryl ammonium bromure (CTAB) $2 \times 10^{-5} \mathrm{M}$ ), d'où elles sont tirées à une vitesse constante de $1 \mathrm{~mm} / \mathrm{min}$ [11].

Le CTAB ainsi déposé sur les lames induit une bonne homéotropie. La couche conductrice d'oxyde d'indium recouvrant les lames a été préalablement gravée de manière à former dix électrodes linéaires parallèles de $40 \mu$ de large espacées de $60 \mu$ qui forment les lignes et les colonnes de la matrice.

2. Diffraction par la matrice. - La matrice placée dans un faisceau laser, produit une image de diffraction qui varie avec la tension appliquée. Cette image correspond à la figure de diffraction du point élémentaire échantillonnée en rapport avec la répétition de ce point dans la cellule.

L'affichage de figures simples (lignes, colonnes, grille) induit seulement une faible modulation des taches de diffraction. Mais l'image de diffraction change fortement suivant que les colonnes voisines sont dans le même état ou non.

Le point élémentaire correspondant à l'intersection d'une ligne et d'une colonne est un objet de phase que l'on peut décrire par $U(x, y)$. Ce motif élémentaire est répété régulièrement dans la cellule.

En chaque point est appliquée une tension correspondant à celle qui est recueillie au point homologue de la matrice de $\mathbf{P}$. Alais. Cependant pour un point donné, $U(x, y)$ ne dépend pas seulement de la tension qui lui est appliquée mais aussi de celle qui est appliquée aux points voisins. La taille des électrodes ainsi que leur espacement doivent être choisis de façon à diminuer ces interactions.

3. Observation microscopique du basculement. Observée au microscope, en lumière blanche et entre polariseurs croisés, une cellule d'épaisseur variable, constituée d'une lame sur laquelle sont gravées des électrodes parallèles de $40 \mu$ de large et une lame non gravée permet d'étudier le mode de basculement des molécules. La direction des électrodes de la lame gravée est parallèle à l'une des directions de polarisation.

Quand la tension appliquée à la cellule est supérieure à la tension seuil nécessaire pour faire basculer les molécules, une ligne sombre apparaît au milieu des électrodes. Lorsque l'on fait tourner la cellule, cette ligne, qui donne le lieu des molécules qui ont basculé parallèlement à une des directions de polarisation, se déplace vers un bord de l'électrode alors qu'une ligne similaire naît à l'autre bord.

Le repérage de la direction du grand axe des molécules [12] permet de définir leur disposition sous l'électrode (Fig. 1).

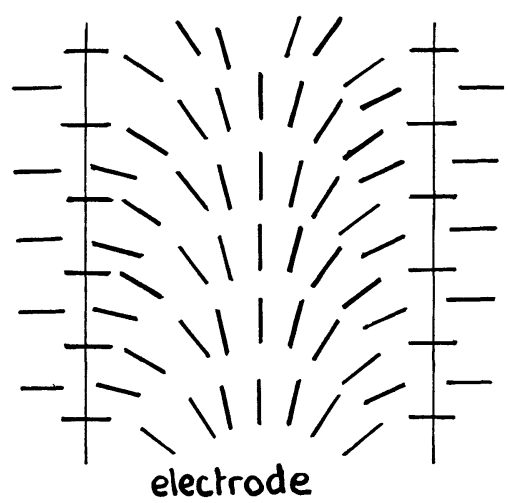

FIG. 1. - Directions de basculement des molécules sous une électrode de faible largeur $(40 \mu)$.

On remarque que les molécules basculent en dehors des électrodes à cause des effets de bords d'origine électrique. Les zones perturbées entre deux électrodes peuvent se rejoindre si la tension appliquée est suffisamment importante.

Afin de minimiser ces effets nous avons essayé de déterminer la largeur et l'écartement des électrodes les plus favorables compte tenu de la commande électrique choisie. Nous avons étudié l'extension des effets de bord en fonction de l'épaisseur de la cellule et la largeur des électrodes. Les résultats sont résumés sur les figures $3 a$ et $b$. L'extension des effets de bords sous tension sinusoïdale constante $(f=1500 \mathrm{~Hz})$ est une fonction linéaire de l'épaisseur de la cellule ; la figure $3 b$ indique pour une épaisseur donnée l'espacement minimum au-dessous duquel il y a recouvrement des zones basculées à l'extérieur de deux électrodes voisines.

Une cellule matricielle donne lieu à des défauts, appelés ombilics (Fig. 2a) [13, 14]. Observés entre polariseurs croisés, ce sont des croix noires dont les branches correspondent à une région où la projection de l'axe optique sur un plan parallèle aux lames est selon l'une ou l'autre des directions de polarisation.

$\mathrm{Au}$ croisement d'une ligne et d'une colonne ou d'un espace interligne et d'un espace intercolonnes, ces ombilics sont de type - 1, la croix noire tourne dans le même sens et deux fois plus vite que la cellule. Ailleurs les ombilics sont de type +1 (Fig. $2 b$ ).

Lorsque l'on affiche une image sur la matrice, les différentes colonnes (lignes) ne sont pas toutes au même potentiel. Etant donné le faible écartement entre deux électrodes voisines il y a entre celles-ci des interactions qui déforment les points.

Ces données conjuguées au souci d'obtenir des points relativement homogènes tout en limitant les interactions entre électrodes voisines permettent de déterminer les paramètres favorables concernant les dimensions physiques de la matrice.

Ainsi, pour les conditions d'attaque choisies et pour une cellule d'une épaisseur typique de $10 \mu$, des électrodes de $100 \mu$ espacées de $80 \mu$ semblent bien adaptées. Pour l'affichage d'un hologramme acoustique ces 


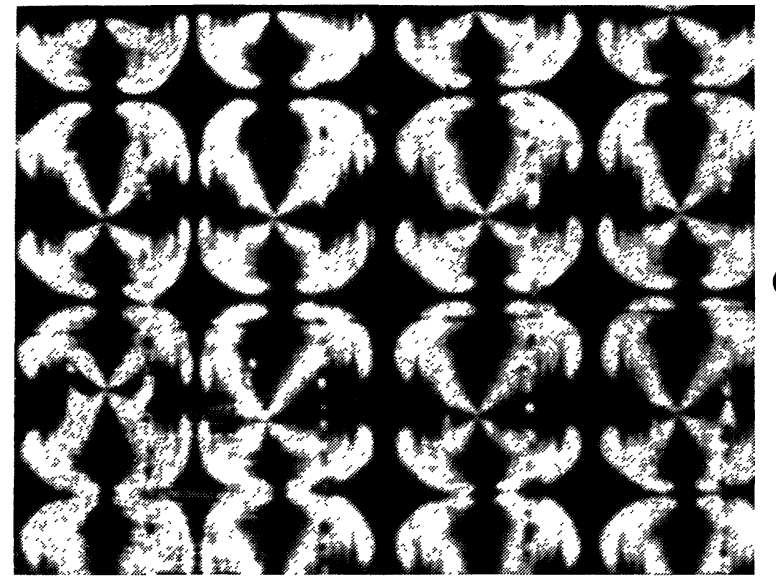

Fig. 2a. - Aspect des ombilics pour une cellule matricielle à électrodes étroites.

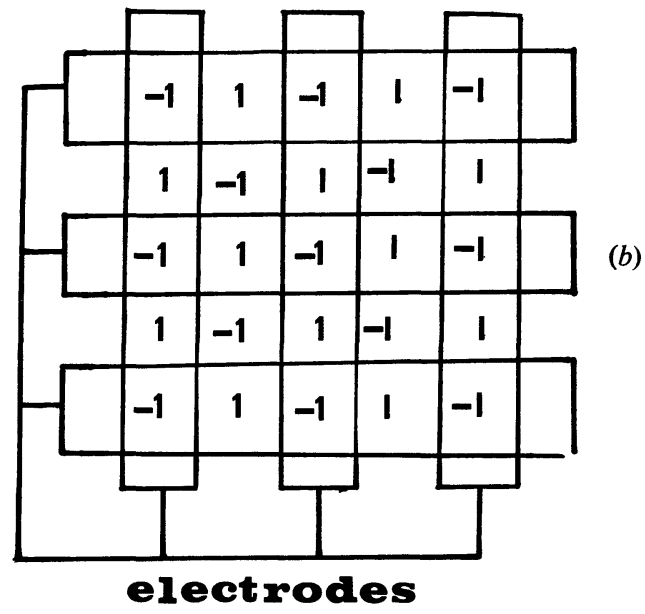

FIG. $2 b$. - Classification des ombilics.

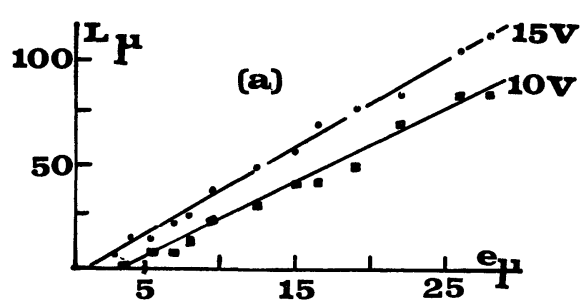

Fig. 3a. - Extension $L$ de la zone perturbée par les effets de bord en fonction de l'épaisseur $e$ de la cellule.

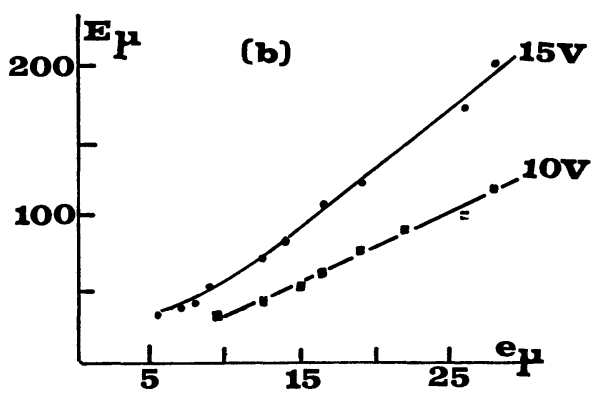

FIG. $3 b$. - Ecartement $E$ de deux électrodes voisines pour lequel il y a recouvrement des effets de bord, en fonction de l'épaisseur $e$ de la cellule. dimensions permettent une réduction de taille du hologramme d'un facteur 15 [4, 5]. Il est possible d'augmenter ce facteur en utilisant une cellule d'épaisseur plus faible (Fig. 3).

4. Affichage du hologramme. - Il nous faut obtenir une modulation de phase convenable (il semble que pour un hologramme de phase une excursion de $\pi / 10$ à $\pi / 5$ soit particulièrement favorable), et ce avec un scintillement très faible puisque nous affichons un hologramme et non une image ; ce scintillement sera réduit en formant l'image très rapidement.

Les $n$ lignes de la matrice sont adressées séquentiellement par une tension $m V$; les colonnes, attaquées en parallèle reçoivent $+V$ ou $-V$ selon qu'elles correspondent sur la ligne adressées à un point éteint ou allumé (Fig. 4a), le point en question ayant alors à ses bornes $(m-1) V$ ou $(m+1) V$. En dehors de la ligne adressée tous les points voient $\pm V$ mais l'effet n'est sensible qu'à la valeur efficace.

(a)

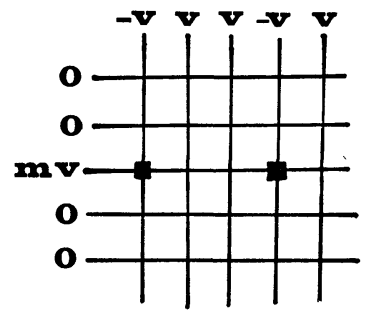

Fig. 4a. - Schéma d'application des tensions. Les lignes sont adressées par le signal « $m V \Downarrow$, les colonnes sont attaquées en parallèle par « $+V$ » ou «-V» selon que le point correspondant de la ligne adressée est éteint ou allumé.

(b)

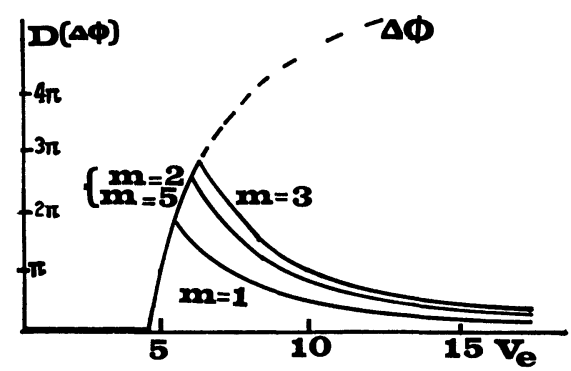

Fig. 4b. - Différence de déphasage entre un point éteint et un point allumé $-n=10$ lignes pour divers valeurs de $m$ en fonction de la tension efficace appliquée au point allumé.

Un point allumé est soumis à une tension $(m+1) V$ pendant le temps $t$ correspondant à l'adressage de la ligne et à une tension $V$ pendant $(n-1) t$ correspondant à l'adressage des autres lignes.

La valeur efficace de la tension reçue est donc pour un point allumé

$$
\sqrt{\frac{(m+1)^{2} V^{2}+(n-1) V^{2}}{n}}
$$


c'est-à-dire

$$
V \times \sqrt{\frac{m^{2}+2 m+n}{n}}
$$

Pour un point éteint la tension efficace est

$$
V \times \sqrt{\frac{m^{2}-2 m+n}{n}}
$$

Le rapport des tensions est donc

$$
R=\sqrt{\frac{m^{2}+2 m+n}{m^{2}-2 m+n}}
$$

ce rapport est maximum pour $m=\sqrt{n}$, c'est alors que l'on peut avoir un écart de phase maximum entre un point éteint et un point allumé. Mais pour $n$ grand nous ne pourrons peut-être pas utiliser le $m$ optimal qui, important, peut occasionner des interactions entre ligne adressée et lignes voisines.

Nous avons appliqué à une cellule un signal $(m+I) V$ ou $(m-I) V$ durant $t$ et $V$ durant $(n-I) t$ afin de simuler un point de la matrice.

La différence de déphasage entre un point allumé et éteint correspond à ce que l'on voit en figure $4 b$.

La courbe de déphasage $\Delta \varphi$ en fonction de la tension efficace $V_{\mathrm{e}}$ appliquée à un point allumé est représentée en pointillé. Pour un point éteint, la courbe de déphasage se déduit de la précédente par une homothétie selon l'axe des tensions.

Le rapport d'homothétie est $R$, rapport des valeurs moyennes des tensions entre un point allumé et un point éteint.

On a représenté sur la figure la différence de déphasage, $D(\Delta \varphi)$ entre un point allumé et un point éteint en fonction de la tension pour diverses valeurs de $m$.

Pour des tensions élevées nous avons encore observé un écart de phase proche de $\pi / 2$ pour une épaisseur de cales de $8 \mu$ et $n=256$ lignes $m=8$, ce qui est donc très satisfaisant. Le cristal liquide employé étant du M. B. B. A.
Pour 256 lignes, le scintillement est encore nul pour une cellule de $15 \mu$ d'épaisseur et une période image de $5 \mathrm{~ms}$ (et certainement bien plus). Toutefois les temps de commutation entre l'état allumé et l'état éteint sont très longs (de l'ordre de la seconde).

Une cellule de $8 \mu$ a des temps de commutation plus faibles $(100 \mathrm{~ms})$ mais un scintillement apparaît pour une période image de $2,5 \mathrm{~ms}$. Ce scintillement croît avec $m$ et avec le déphasage.

Une étude optique permettra de déterminer la modulation de phase souhaitable et le scintillement supportable.

5. Conclusion. - Nous avons étudié une matrice à cristaux liquides pour la reconstitution d'un hologramme acoustique.

Nous avons choisi des électrodes de très petites dimensions afin de réduire les distorsions lors de la reconstitution optique; ces faibles dimensions ont conduit à l'affichage d'une image de phase d'un traitement optique difficile. Une étude systématique a permis de connaître les dimensions réduisant les interactions responsables de ce mauvais fonctionnement tout en restant raisonnablement faibles pour une bonne reconstitution.

Par ailleurs nous obtenons sur le plan de l'affichage une modulation de phase suffisante. Le scintillement doit être très réduit, étant donné l'utilisation holographique, ceci limite dans l'immédiat le dispositif à la visualisation d'images statiques ou à l'utilisation en amont de transducteurs acousto-électriques assurant une exploration très rapide du hologramme acoustique.

La possibilité de créer électriquement un hologramme de phase, fût-il statique est cependant déjà très intéressante sur le plan optique.

Remerciements. - Nous remercions M. le Professeur Alais pour la lecture critique du manuscrit et MM. Schuller et Fruchard pour leur aide technique.

\section{Bibliographie}

[1] Mueller, R. K. and Keating, P. N., Acoustical Holography (Plenum Press, New York)1969, vol. I, p. 49 H. M. A. EL-SUM page 1.

[2] Fox, M. D., Thurstone, F. L., Von Ramm, O. T., Acoustical Holography (Plenum Press, New York) 1972, vol. 4, p. 504.

[3] Alais, P., Acoustical Holography (Pelnum Press, New York) 1972, vol. 4, p. 237.

[4] Kreuzer, J. L., Acoustical Holography (Plenum Press New York) 1969, vol. I, p. 86.

[5] Clement, M. and Griffiths, J. W. R., Non destructive testing 6 (1973) 175-222.

[6] Lechner, B. J. L., Marlowe, F. J., Proc. IEEE 59 (1971) 1566.
[7] Assouline, G., Electron. Lett. 8 (1972) 45.

[8] Bigelow, J. E., Kashnow, R. A., Steim, G. R., I. E. E. E. Trans. Elec. Dev. ED 21146 (1974).

[9] Bigelow, JE. E., Kashnow, R. A., Steim, G. R., I. E. E. E. Trans. Elec. Dev. Janv. (1975).

[10] Labrunie, G., Robert. J., Borel, J., Revue Phys. Appl. 10 (1975) 143.

[11] Proust, J. E., Terminassian-Saraga, L., Guyon, E., Solid State Commun. 11 (1972) 1227.

[12] Mauguin, C., C. R. Hebd. Séan. Acad. Sci. 154 (1912) 1359.

[13j Rapini, A., Leger, L. and Martinet, A., J. Physique Colloq. 36 (1975) C1-189.

[14] Rapin : A., J. Physique 34 (1973) 629. 